\title{
La política educativa como negocio. Ajuste presupuestario, discurso meritocrático y el "davos" de la educación en Argentina (2015-2019)
}

\author{
Educational policy as business. Budget adjustment, meritocratic discourse and the "davos" of \\ education in Argentina (2015-2019)
}

\section{A política educativa como negócio. Ajuste do orçamento, discurso meritocrático e o "davos" da educação na Argentina (2015-2019)}

Estela Maria Miranda*

\section{Resumen}

El propósito de esta ponencia es identificar algunos "indicadores del imaginario neoliberal" (BALL, 2011) en las políticas educativas implementadas en la gestión de la Alianza Cambiemos, a partir de diciembre de 2015, analizando los avances en la privatización de la educación mediante el rediseño de las relaciones entre sector público/privado a través de una variedad de asociaciones públicas y privadas (BALL; YOUDELL, 2007; RIZVI; LINGARD, 2013). En ese marco el discurso de la meritocracia construye un sentido común que sirven como justificación para la introducción de herramientas de la Nueva Gestión Pública (NGP) en las políticas del gobierno de Cambiemos. Posteriormente, se caracteriza el papel de una variedad de nuevos (y otros no tan nuevos) "participantes híbridos" que intervienen crecientemente en el diseño y ejecución de las políticas educativas. Sobre resultados de investigaciones en curso se toman como referencia casos o situaciones para ilustrar los análisis y luego trazar un balance provisorio.

Palabras-Clave: Neoliberalismo. Políticas Educativas. Privatización. Nueva Gestión Pública.

\section{Abstract}

The purpose of this paper is to identify some"indicators of the neoliberal imaginary"(BALL, 2011) in the educational policies implemented in the management of the Cambiemos Alliance, as of December 2015, analyzing the progress in the privatization of education through the redesign of public/private sector relationships through a variety of public and private partnerships (BALL; YOUDELL, 2007; RIZVI; LINGARD, 2013). Within that context, the meritocracy discourse builds a common sense about realities that justify the introduction of New Public Management (NGP) tools in the Cambiemos government policies. Subsequently, the role of new (and not-sonew) "hybrid participants" that are increasingly involved in the elaboration and implementation of educational policies is characterized. On the results of ongoing investigations, cases or situations are taken as references to illustrate the analyzes and then draw up a provisional balance.

Keywords: Neoliberalism. Educational Policy. Privatization. New Public Management.

Recebido em 04/05/2019 - Aprovado em 16/09/2019

http://dx.doi.org/10.5335/rep.v27i1.10572

Doctora en Ciencias de la Educación en Universidad Nacional de Córdoba (UNC, Argentina). Profesora Titular de Política Educacional y Legislación Escolar. Directora del Doctorado en Ciencias de la Educación (2002-2013) (UNC, Argentina). ORCID https://orcid.org/ 0000-0002-8499-8888. E-mail: estelam@ffyh.unc.edu.ar 


\section{Resumo}

O propósito deste artigo é identificar alguns "indicadores do imaginário neoliberal" (BALL, 2011) nas políticas educativas implementadas na gestão da Alianza Cambiemos, a partir de dezembro de 2015, analisando os avanços na privatização da educação mediante o redesenho das relações entre setor público/privado através de uma variedade de associações públicas e privadas (BALL; YOUDELL, 2007; RIZVI; LINGARD, 2013). Neste cenário, o discurso da meritocracia constrói um sentido comum que serve como justificativa para a introdução de ferramentas da Nova Gestão Pública (NGP) nas políticas do governo de Cambiemos. Posteriormente, se caracteriza o papel de uma variedade de novos (e outros não tão novos) "participantes híbridos" que intervêm crescentemente no desenho e execução das políticas educativas. Sobre resultados de investigações em curso se tomam como referência casos ou situações para ilustrar as análises e em seguida traçar um balanço provisório.

Palavras-chave: Neoliberalismo. Políticas Educativas. Privatização. Nova Gestão Pública.

La educación pública es un sistema abierto a todo el mundo sin discriminaciones por motivos de género, religión, cultura

o clase social, gratuito, financiado por el sector público, y gestionado y evaluado conforme a los objetivos y principios establecidos democráticamente por las autoridades públicas.

(V Congreso Mundial. Internacional de la Educación. Porto Alegre 2004).

\section{Introducción}

El análisis de políticas educativas en tiempos de un fuerte avance del neoliberalismo autoritario en la región es una tarea altamente necesaria aunque no sencilla.

Interpretar las políticas educativas locales y su vinculación con los movimientos globales es una forma de visibilizar lo que defino como procesos subterráneos que están aconteciendo en nuestros países y se han instalado en el sentido común de las escuelas, los docentes, los padres y de la sociedad toda. La magnitud y el rápido avance de la mercantilización y privatización de la educación interpela a la producción de conocimientos para desentrañar los discursos que circulan y las prácticas que están transformando profundamente los modos como pensábamos hasta ahora los sistemas educativos nacionales.

En los últimos años los estudios sobre privatización y mercantilización de la educación fueron ganando centralidad en las agendas de investigación y en los debates sobre las políticas educacionales. Es importante destacar que disponemos de un caudal de resultados y referencias analíticas sobre estos procesos en otras realidades investigadas, que aportan categorías fértiles a nuestras investigaciones. Sin embargo, persisten dificultades del orden de lo metodológico y, sobre todo, vinculadas al acceso a fuentes de datos para hacer "etnografía de redes políticas" 
que requiere de un intenso y complejo proceso para rastrear esa compleja trama de nuevos actores con modos de vinculación, articulaciones y relacionamientos diversos y complejos (BALL, 2014, p. 28).

Respecto de la información oficial, que sería esperable encontrar en el sitio web del Ministerio de Educación en Argentina, se observa una alta precariedad y dispersión en las estadísticas educativas y, de modo muy general, algunos datos sobre las acciones que desarrolla ese organismo. La falta de transparencia sobre la gestión pública, se agudiza porque fueron cambiados o modificados los sitios web del gobierno anterior y buena parte de la información se perdió. Lo que implica claramente una política de desinformación que, además, restringe las posibilidades de consulta de fuentes necesarias para la investigación y la enseñanza sobre las políticas educativas recientes (VISACOVSKY, 2019).

El propósito de esta presentación es identificar algunos "indicadores del imaginario neoliberal" (BALL, 2011) en las políticas educativas implementadas en la gestión de la Alianza Cambiemos, a partir de diciembre de 2015, analizando los avances en la privatización de la educación mediante el rediseño de las relaciones entre sector público/privado a través de una variedad de asociaciones públicas y privadas (BALL; YOUDELL, 2007; RIZVI; LINGARD, 2013). En ese marco el discurso de la meritocracia construye un sentido común que sirve para justificar la introducción de herramientas de la Nueva Gestión Pública (NGP) en las políticas del gobierno de Cambiemos. Posteriormente, se caracteriza el papel de una variedad creciente de nuevos (y otros no tan nuevos) "participantes híbridos" que intervienen crecientemente en el diseño y ejecución de las políticas educativas. Sobre resultados de investigaciones en curso se toman como referencia casos o situaciones para ilustrar los análisis y luego trazar un balance provisorio.

\section{Poniendo la política educativa en contexto (2015-2019). De la producción de sujetos de derechos a sujetos consumidores.}

Comojustificativo de la "modernización" de la educación, la Alianza Cambiemos que asume el gobierno nacional en diciembre 2015 instala el discurso de la "refundación" de la educación en Argentina, a partir de un fuerte cuestionamiento de las políticas educacionales del gobierno anterior.

Cabe recordar que los gobiernos nacionales, a partir de Mayo de 2003, recuperando la marca de origen de la educación pública, sancionaron un conjunto de leyes ${ }^{1}$ que tuvieron como propósito recomponer la centralidad del Estado en la formulación y ejecución de las políticas educativas y dejar planteado de modo 
contundente en el texto de la Ley de Educación Nacional que la educación es un derecho humano, público y social. Para efectivizar ese derecho ${ }^{2}$ se diseñó un ensamble de políticas sociales y educativas, en una clara ruptura con el discurso neoliberal y la visión mercantilista de la educación de la década de los noventa (MIRANDA, 2013, p. 26).

El gobierno actual, con una participación muy activa de los medios de comunicación hegemónicos y de referentes de la cultura, fue instalando "un sentido común" en la sociedad sobre el deterioro de la calidad de la educación pública asociada a las políticas educativas de reconocimiento de derechos del gobierno anterior. En los discursos de los diferentes funcionarios, incluido el Presidente, expresiones metafóricas como: "llenar de universidades públicas toda la provincia cuando todos sabemos que nadie que nace en la pobreza llega a la universidad?" se escucharon de la actual gobernadora de la provincia de Buenos Aires, en referencia crítica a las universidades nacionales ${ }^{3}$ creadas durante la gestión de Cristina Fernández de Kirchner (EL PAÍS DIGITAL, 18). Recientemente el Presidente de la Nación sostenía: "De qué servía repartir computadoras si no tenían conexión a Internet. Es como repartir asado y no tener parrilla, ni algo para prender el fuego", para desacreditar el Programa Conectar Igualdad4 (CLARÍN, 22/05/19). En medio del conflicto salarial docente en marzo de 2017, el Presidente en conferencia de prensa dio a conocer los resultados del Operativo Aprender ${ }^{5}$, comparando y destacando los resultados aventajados obtenidos en las evaluaciones por los alumnos que asisten a escuelas de gestión privada, para concluir con una fuerte estigmatización de la escuela de gestión pública: "Una terrible inequidad de aquél que puede ir a la escuela privada versus aquél que tiene que caer en la escuela pública”. En el discurso de Cambiemos a la educación pública no se llega por elección, se cae por descarte, porque no queda otra. En la educación pública "caen" los sectores carenciados que no pueden comprar o pagar por escuela privada, eso es lo que se interpreta como una "terrible inequidad" para estos sectores, sin mencionar la responsabilidad que corresponde al Estado de garantizar el "derecho de aprender" establecido en la Constitución de la Nación Argentina desde 1856 y en la Ley de Educación Nacional (2006) cuando en el artículo $2^{\mathrm{a}}$ establece: La educación y el conocimiento son un bien público y un derecho personal y social garantizado por el Estado.

Otro testimonio que da cuenta del lugar que ocupa la educación pública en las prioridades del gobierno de Cambiemos se revela cuando analizamos la inversión educativa. Un estudio publicado por Confederación de Trabajadores de la Educación 
de la República Argentina (CTERA) señala que a partir de 2016 el gobierno nacional inició un proceso de ajuste en la inversión educativa, con un reducción del 5\% en términos reales. Ese proceso de desfinanciamiento ${ }^{6}$ fue sólo el reflejo en el plano presupuestario del desmantelamiento de planes y programas educativos. En 2017 continúa el ajuste y la subejecución presupuestaria, un procedimiento financiero muy usado por este gobierno. Lo mismo para 2018 con un mayor recorte en programas socioeducativos, becas, ampliación de jornada extendida, formación docente y distribución de equipos informáticos. El Informe de la CTERA denuncia la situación y manifiesta su preocupación por el incumplimiento de la meta del 6\% del PBI (Producto Bruto Interno) destinado a educación, se acuerdo a lo establecido por la Ley de Financiamiento Educativo (2005) y la Ley de Educación Nacional (2006). Es importante destacar que con el anterior gobierno, en 2015, la inversión había alcanzado un 6,9\% del PBI (CTERA, 2018, p. 4). Estudios realizados por la Universidad Nacional Pedagógica (UNIPE) indican que la inversión por alumnos se redujo en alrededor de un $20 \%$ entre 2016 y 2019 , no obstante, fue significativo el aumento en la partida para evaluaciones educativas (GOROSTIAGA, 2019, p. 3).

La educación se concibe como un bien de consumo privado que se puede conseguir con el esfuerzo individual. Los derechos consagrados en la legislación se asocian a una concepción liberal de igualdad de oportunidades que no reconoce las diferencias y desigualdades en las trayectorias de vida de los sujetos que ingresan al sistema educativo. Para la ideología neoliberal y neoconservadora de la Alianza Cambiemos, la educación como derecho social, resultado de luchas colectivas, va en desmedro de la "iniciativa individual y privada".

Para Laval, en la lógica racional neoliberal "cada individuo debe comportarse como una especie de empresa situada en el mercado" (2018, p. 40). Desde una concepción meritocrática el éxito es el resultado del talento más esfuerzo individual para ganarse o merecer lo logrado. En su etimología, meritocracia proviene del latín meritum. Sistema basado en el mèrito. Participio pasivo de mereri, ganar, merecer. Acto que hace a una persona digna de galardón o de sanción. También lo que justifica un fracaso. En el discurso de la meritocracia: talento + esfuerzo es la ecuación perfecta. Por tanto, el éxito o el fracaso es asunto de cada uno, mérito propio y no también del modelo económico y del rol que asume el Estado. La responsabilización y la culpabilización recae en los sujetos.

Porque las personas también se deben gestionar como una empresa privada. El mérito individual está asociado a inventarse a sí mismo, el "emprendedurismo es el modelo del éxito". Parecería que las condiciones contextuales no intervienen, 
no tienen nada que ver con el éxito o el fracaso individual. No se me ocurre cómo con las tasas siderales de interés que cobran los bancos para obtener un crédito, hoy en Argentina, se podría generar una cultura de la inversión y del emprendedurismo. Por mejores ideas que tenga un emprendedor ¿Dónde obtiene el capital/recursos para iniciar la actividad? Como señala Thomas Piketty,

El neoliberalismo ha demostrado no ser un buen sistema de distribución de la riqueza ya que no recompensa el trabajo -y por tanto a los trabajadores quienes obtienen sus rentas del trabajo-, sino que favorece a quienes ya poseen la riqueza heredada, los rentistas (GARCÍA DELGADO; GRADIN, 2017, p. 27).

Ideas como "Refundar la educación", la "revolución educativa", "formar emprendedores", "formar para la incertidumbre y disfrutarla" se pueden interpretar como poner a la educación al servicio de la economía y del empresariado. En el Documento oficial Educación y trabajo: Diagnóstico, se destacan tres tendencias relevantes que afectan el mercado laboral a nivel global: a) el desplazamiento productivo y geográfico de los sectores tradicionales(manufacturas servicios de bajo valor agregado) hacia sectores nuevos (agroindustria, servicios intensivos en conocimiento); b) La automatización, es decir la creciente complementariedad de tecnología y empleo calificado y sustitución de capital por empleo; c) El creciente cuentapropismo que incluye modalidades de demanda (tipo Uber o AirBnB) part time o por períodos determinados, que requiere de cambios en los regímenes laborales vigentes. Como en el mismo documento se menciona se trata de generar "un marco regulatorio del empleo" "una nueva y difusa relación laboral" que permita aumentar la maleabilidad, movilidad y capacidad e incorpore nuevas figuras de contratación (independientes, trabajadores por demanda, etc). En otros términos, se trata de trabajo precario, aumento de la informalidad, flexibilización laboral en una reforma laboral con la consiguiente pérdida de derechos laborales, salariales y de seguridad social (PRESIDENCIA DE LA NACIÓN, JEFATURA DE GABINETE DE MINISTROS, 2017, p. 4; AMAR, 2019, p.3).

Desde el inicio de la gestión y a través de el primer Ministro de Educación nacional, Esteban Bullrich, las orientaciones del sistema educativo a las demandas del economía y del empresariado se plasman, como vimos, en documentos oficiales y en las expresiones metafóricas utilizadas en diferentes contextos (discursos en reuniones y foros empresariales, artículos y entrevistas en medios de comunicación). En un nota periodística el entonces Ministro Bullrich refiriéndose al mercado laboral señalaba: 
Vivimos en un mundo en el que se están creando nuevos trabajos todos los días. Un chico de hoy tendrá a lo largo de su vida siete empleos, cinco de los cuales aún no fueron creados. No podemos preparar a los chicos hoy para los empleos que vendrán, pero podemos formarlos para dos cosas: para que aprendan a disfrutar de esta incertidumbre y para que sean los que salgan a crear esos empleos (LA NACIÓN, 21/07/2016)

En oportunidad de la $52^{\circ}$ reunión anual de las empresas más grandes del país en el Coloquio de IDEA, el 16 de Octubre de 2016, el Ministro de Educación participó del panel "De la educación al trabajo: cómo vamos a llegar" enfatizando en la necesidad de ir hacia un mundo "donde el sistema educativo pueda desarrollar los talentos individuales", para luego señalar que "No sirve más el sistema educativo argentino. Está diseñado para hacer chorizos, todos iguales. Se diseñó para tener empleados en una empresa que repetían una tarea todo el día, que usaban el músculo y no el cerebro y nunca lo cambiamos. Queremos que los jóvenes salgan a crear trabajo no a buscarlo". Desde la perspectiva de Cambiemos el talento es algo que nos viene dado por naturaleza y el capital económico que el emprendedor necesita para hacer efectiva su idea también. En el planteo del Ministro no se explica cómo participará el Estado en el diseño y sostenimiento de un nuevo sistema educativo donde los alunnos puedan adquirir las capacidades para el "uso del cerebro" que demandan los puestos laborales aún no creados. Tampoco dice cómo el Estado participa en la creación de créditos para apoyar los emprendimientos (ya sea para manejar drones o para hacer emprendimientos de cerveza artesanal como recomendo en varias oportunidades el Ministro)

De regreso del Foro de Davos, en 2018, en el que participó como Senador de la Nación, escribió una nota publicada en el Diario La Nación en la que retoma uno de los ejes del Foro: el futuro del empleo,

El tema fue recurrente no solo en los paneles, sino además en los discursos de los líderes políticos y empresariales (Jack Ma y Bill Gates, por nombrar algunos). La otra palabra de moda fue reskilling (recapacitarse o reformarse): la práctica de ayudar a trabajadores que han perdido el empleo o corren riesgo de perderlo a adquirir las competencias y/o habilidades necesarias para no salir del mundo del trabajo (LA NACIÓN, 2018).

En otro momento de la nota y colocándose en el lugar de las responsabilidades políticas y empresariales sostiene: "Es, sin lugar a dudas, el desafío más importante que tenemos dirigentes políticos y empresariales para los próximos años. Garantizar a nuestros ciudadanos una educación de por vida que les permita en cualquier momento adquirir las nuevas habilidades necesarias para encontrar un trabajo digno". Entre las recomendaciones que surgieron del Foro de Davos Bullrich destaca?: 
1. Acuerdos público-privados. La tarea de reskilling no puede ni debe ser solo estatal. Incluyendo la participación de los empresarios y los sindicatos. 2. Formación en habilidades blandas para todos y todas. Es fundamental que los ciudadanos activos, estén o no empleados, tengan la posibilidad de incorporar las llamadas habilidades blandas: creatividad, trabajo en equipo, expresión oral y escrita, empatía, por citar algunas. Todas necesarias en el mundo laboral de la cuarta revolución industrial. 3. Reskilling para los maestros. Todos estos cambios deben llegar a las escuelas de nuestro país. Es decir, a nuestros maestros y maestras. Esa es la única garantía que tenemos de no repetir una situación de este tipo. Continuar el trabajo en la formación docente es clave para que nuestros jóvenes dominen la revolución tecnológica y sean ciudadanos plenos en el mundo actual (LA NACIÓN, 2018).

En definitiva, cuando el Presidente Macri habla de "Refundar la educación" o la "revolución educativa", quiere decir sustituir a la escuela pública, que es lo constitutivo de nuestra identidad nacional, una pieza clave en la conformación del Estado-Nación, un ámbito de socialización, integración e inclusión educativa y un poderoso vehículo para la movilidad social ascendente, altamente valorada en el imaginario colectivo de Argentina. En el pensamiento del gobierno actual el problema está en la gestión pública porque el supuesto es que la gestión privada (educación, salud, servicios en general, etc.) garantiza mejor calidad, sin conflictos, por tanto, es preferible que se ocupen otros proveedores del servicio educativo mientras el Estado actúa como garante produciendo normas de mercado que "regulan a distancia" la competencia entre individuos y organizaciones

\section{Del gobierno a la gobernanza. Rendición de cuentas (RdC) como herramienta de gestión de políticas educativas.}

Los contextos nacionales particularmente en la región, pero también en otras partes del mundo, están asistiendo a un conjunto de "movimientos" de modernización de los servicios públicos, de los aparatos estatales y la arquitectura total de los Estados en diferentes escalas de operaciones (BALL, 2013, p.177). Lo que está ocurriendo sostiene el sociólogo inglés es un:

Traspaso hacia un Estado policéntrico y un desplazamiento del centro de gravedad en torno al que giran los ciclos de la política que incrementan la gama de actores que participan del diseño y aplicación de las políticas. Este cambio se suele describir como un cambio de perspectiva: del gobierno a la gobernanza, o a la gobernanza en red, o el paso al estado posburocrático o "lean government" (gobierno limitado a costo eficiente), que implica el desarrollo de relaciones que suponen reciprocidad e interdependencia en contraposición a jerarquía e independencia (BALL, 2011, p. 28)

Desde una perspectiva foucaultiana, Christian Laval sostiene que "la racional neoliberal" se apoya en "Un sistema de normas o una lógica general que impone 
el modelo de mercado, en todos lados, por el cual los individuos y las instituciones son puestos en competencia, de manera que cada entidad, cada individuo debe comportarse como una especie de empresa situada en el mercado". El Estado es el "principal agente", según Laval, y su actuación es determinante para la generación de "un conjunto de normas de mercado en la sociedad, instituciones e individuos, a la que trata de reformar" (2018, p. 40).

En los movimientos de modernización de los servicios públicos, Robert Jessop destaca que se está produciendo un proceso de "desestatización" que

Implica redefinir la separación público-privado, redistribuir tareas y rearticular las relaciones entre organizaciones y tareas por medio de esa separación. Esta redefinición y redistribución tiene varios aspectos, algunos viejos otros más nuevos, como la creación de órganos ejecutivos, o el establecimientos de asociaciones público-privado (de muchos tipos diferentes), contratación de proveedores privados para que realicen servicios estatales o contratación de think tanks (laboratorios de ideas), consultores y empresas especializadas en conocimiento para realizar diagnósticos, investigaciones, evaluaciones de políticas (BALL, 2013 p. 177).

En otras palabras, lo que antes hacia el Estado ahora es realizado por esos otros; "nuevas voces e intereses están representados en el proceso político, nuevos nudos de poder e influencia son construidos y fortalecidos" (BALL, 2013, p. 177) y "la comunidad que constituye la política educativa es cada vez mayor" (BALL, 2011, p. 2).

Entonces, el Estado no se tiene que ocupar de gestionar los servicios educativos para garantizar derechos fundamentales como la educación a los ciudadanos. El Estado tiene la función de "gerente", utilizando herramientas de gestión que provienen de la Nueva Gestión Pública (NGP) (New Public Management)

Las reformas promovidas por la NGP buscan generar rediseños organizacionales en instituciones del sector público para lograr mayores niveles de eficiencia y promover su calidad, todo ello basado en prácticas ya existentes en el sector privado, es decir, en la empresa privada que es el modelo a seguir en todos los aspectos de la vida de los países y de las personas (MIRANDA; SALTO, 2015, p. 278).

Para Verger y Parcerisa la política de Rendición de Cuentas (RdC)

Representa un tipo de reforma educativa atractivo para muchos gobernantes ya que, por regla general, permite a los gobiernos transmitir a los votantes -y a la ciudadanía en general- el mensaje de que están trabajando arduamente para el cambio educativo, y para la mejora de los resultados de aprendizaje y de las oportunidades futuras de los más jóvenes. Al mismo tiempo, las reformas de RdC tienden a implicar un bajo riesgo político ya que, en gran medida, la presión de la reforma recae en las escuelas y en los maestros, y no tanto en el gobierno (2018, p. 66). 
Desde la lógica de la NGP para la Alianza Cambiemos el rediseño del sistema educativo está dirigida atender la demanda de los sectores empresariales, en dos sentidos. Por una parte, la formación de capital humano para la "incertidumbre laboral y disfrutarla" (como propone el ex Ministro Esteban Bullrich) en puestos laborales que todavía no fueron creados. Por otra, dejar a las empresas que hagan inversiones en educación, como propone Sanchez Zinny "formar talentos a pedido de las empresas o bien alentando el compromiso de las empresas para invertir en educación ante el aumento de la demanda de capital humano calificado" (2015, p. 50).

Entonces, el Estado debe generar las normas, "actuando como árbitro", para facilitar la intervención de los empresarios en las innovaciones y en el sostenimiento de la educación en la modalidad "nueva filantropía". En el libro "Educación 3.0: La batalla por el talento en América Latina” el ex director del Instituto de Educación Técnica (INET) y actual Director General de Educación de la pcia de Buenos Aires, sostiene: "Mientras que los empresarios y las compañías impulsan la innovación en la educación, el sector público debe actuar como árbitro y asegurar que la dinámica generada por las fuerzas del mercado se canalice de manera efectiva, coordinada y regulada (SANCHEZ ZINNY, 2015, p. 59).

En su condición de "árbitro" el gobierno de Cambiemos utiliza la evaluación de los aprendizajes como una herramienta para la rendición de cuentas. La Secretaría de Evaluación fue creada por Decreto del Poder Ejecutivo Nacional con fecha 6 de abril de 2016, desconociendo que la Ley de Educación Nacional estabelece la creación de un Consejo Nacional de Calidad de la Educación ${ }^{8}$. Dicha Secretaria fue creada con un considerable presupuesto, que contrasta con los recortes a otras áreas de la educación, lo que da cuenta de la importancia que le asigna este gobierno a la participación en los programas de evaluación internacional como PISA, regional como ERCE $^{9}$ y a los dos operativos nacionales: Operativo Aprender (que reemplaza al ONE) y Operativo Enseñar, como se señaló anteriormente. La evaluación como herramienta de la gestión de políticas para la rendición de cuentas tiene propósitos de control, disciplinamiento y jerarquización de estudiantes y docentes, a la vez que instala en las instituciones una lógica de la competencia por una matrícula de estudiantes que garantice los mejores rendimientos en esos operativos. Es lo que se puede inferir por el modo en que el gobierno utiliza los resultados de los operativos de evaluación, difundiéndolos en los medios de comunicación para ponderar los logros de las escuelas de gestión privada. El Presidente de la Nación decía, en el discurso de apertura de la Asamblea Legislativa, el 1 de marzo de 2018: "Tenemos 
que poder saber cómo le está yendo a la escuela donde van nuestros hijos. Hoy está prohibido por ley que se publiquen los resultados por escuela y eso no tiene sentido. Por eso les pido que este año legislativo avancemos para cambiar esa norma”. La Ley de Educación Nacional (2006) en su artículo 97 establece que la difusión de información sobre las evaluaciones educativas debe resguardar la identidad de los alumnos, docentes e instituciones educativas, a fin de evitar cualquier forma de estigmatización. Se podría interpretar que el Presidente está pidiendo modificar la normativa para hacer público los resultados de los operativos de evaluación, habilitando la construcción de un ranking de escuelas.

En Argentina, en la segunda mitad del siglo XIX, cuando se discutía la incorporación de la educación católica en las escuelas públicas los debates parlamentarios entre liberales y católicos giraban en torno a la imposición de la fe religiosa en la disputa por el control de las "almas". Hoy, dice Susan Robertson, la disputa es con "[...] otros de un nuevo tipo cuya fe en el mercado, el emprendedorismo y las ganancias orientan las políticas educativas". En el discurso de Cambiemos de lo que se trata es romper la alianza Estado-ciudadano para construir un "ciudadano consumidor" (FELFEDBER et al., 2018, p. 6), en este caso, consumidor para un mercado educativo donde "Las familias despliegan entonces estrategias para elegir su escuela y maximizar las oportunidades de sus hijas e hijos, distinguiéndose en función de sus ingresos y contribuyendo así en segmentar el sistema educativo en estratos socio-económicos" (ARMIJO CABRERA, 2019, p. 193).

\section{Las relaciones público/privado y nuevas formas de privatización de la educación. Filantropía empresarial}

La educación de gestión privada no es un fenómeno nuevo en Argentina, lo que es nuevo es la privatización por otros medios, con otros actores y con propósitos muy diferentes a la privatización tradicional. El proceso de "desestatización", al que nos referíamos anteriormente, ha convertido a los Estados nacionales en "Estado gerencial" de las relaciones del sector público/privado. A partir del gobierno de la Alianza Cambiemos se profundiza la tendencia a la privatización de las políticas y de los servicios, a través de la presencia de fundaciones, thinks tanks, empresas y ONGs en la definición e implementación de las políticas educativas.

Algunas viejas y muchas nuevas y diversas relaciones público-privado podrían reconocerse en lo que Ball y Youdell (2007) examinan como "formas de privatización en la educación pública y de privatización de algunas parcelas de la educación pública": 
1) La "privatización en la educación" o "privatización endógena", son "formas de privatización (que) implican la importación de ideas, métodos y prácticas del sector privado a fin de hacer que el sector público sea cada vez más como una empresa y crecientemente comercial" (BALL; YOUDELL, 2007, p. 13). Dentro de esta modalidad de privatización se destacan las reformas de cuasi-mercado que promueven la competencia entre escuelas, las autonomía escolar, la gestión por proyectos, la responsabilidad y los salarios en función de los resultados y la aplicación de herramientas de gerenciamiento en la gestión de las escuelas.

En el "Plan 2016-2021: Argentina enseña y aprende", aprobado por Resolución № 285/2016 del Consejo Federal de Educación, en el eje: Comunidad educativa integrada, define "a la educación como un proceso social que requiere la participación y el compromiso de todos los actores que son parte de ella para su mejora" y propone "Articular iniciativas de otros organismos públicos, de las organizaciones de la sociedad civil y del sector privado para contribuir al cumplimiento de los objetivos nacionales concertados" (MINISTERIO DE EDUCACIÓN Y DEPORTES, 2016, p. 21; GOROSTIAGA, 2019, p. 7). La propuesta de una mayor participación de la comunidad educativa puede ser leída como: a) una mayor intervención de las familias en la toma de decisiones sobre los asuntos de la escuela; b) una mayor intervención de los mercados para ampliar y diversificar la oferta educativa; c) una mayor presión sobre los padres para ejercer la "elección" de escuelas (BALL, 1998, p. 113).

Un testimonio de alianza de la sociedad civil, empresarial y organismos no gubernamentales es el "Proyecto Eutopía. La Escuela se reinventa", integrada por "la Vicaría Episcopal de Educación del Arzobispado de Buenos Aires, la Fundación Telefónica Movistar y Profuturo, y Panorama de la Organización de los Estados Iberoamericanos para la Educación, la Ciencia y la Cultura (OEI). Se define como "un modelo colaborativo, inclusivo e innovador de transformación de la escuela secundaria, que parte de las posibilidades reales de las escuelas y del liderazgo de los directivos". A la fecha la Red Eutopía nuclea "16 escuelas secundarias de la Ciudad Autónoma de Buenos Aires, una comunidad integrada por 41 directivos, 64 docentes en forma directa y más de 800 en forma indirecta, y cerca de 6 mil estudiantes, pero se propone escalar a otras escuelas y regiones" (PROTECTO EUTOPÍA, 2019). 


\section{2) La "privatización de la educación" o "privatización exógena" es} definida como

La apertura de los servicios de educación pública a la participación del sector privado, a través de modalidades basadas en el beneficio económico, así como la utilización del sector privado en cuanto a la concepción, la gestión o la provisión de diferentes aspectos de la educación pública (BALL; YOUDELL, 2007, p. 13).

Sobre esta modalidad, los autores advierten un crecimiento a gran velocidad y con una variedad de actores y propósitos, como la inversión privada filantrópica en educación de las empresas nacionales y multinacionales. Para Sanchez Zinny el deterioro de la educación pública y la ausencia de una reforma por parte del Estado se resuelve incorporando al sector privado al sistema educativo nacional e impulsando "el aumento de la inversión privada filantrópica en los programas educativos". Destaca la intervención de las empresas "multilatinas"10 como Embraer, Bimbo, Vale, Techint y Odebrecht como "las empresas (que) asumen, de hecho, el asunto en sus propias manos en ausencia de esfuerzos por una robusta reforma por parte del gobierno"(SANCHEZ ZINNY, 2015, p. 56-58).

La privatización de la política educativa a través de las contrataciones, tanto por el gobierno nacional y varios gobiernos provinciales, de los servicios de consultoría de expertos extranjeros que han llevado a cabo reformas de la educación exitosas, como Finlandia, Singapur, Francia para reformar la educación y a corto plazo los niños y adolescentes mejoren los magros resultados en las pruebas PISA y Aprender.

\section{Así, en la prensa nacional como Diario Clarín se podía leer:}

[...] Las últimas pruebas Aprender, por ejemplo, mostraron que siete de cada 10 estudiantes salen de la secundaria sin alcanzar un nivel satisfactorio en la disciplina, lo que dificulta su posterior tránsito por la universidad o conseguir un empleo de calidad. ¿Qué pasa que los chicos no están aprendiendo? ¿Dónde están las trabas?

Para eso, están tomando los conceptos más importantes de los métodos utilizados en otros países, como Singapur -que es el de mayor éxito en las pruebas internacionaleso Francia, con una tradición educativa similar a la Argentina y que en estos días está iniciando un ambicioso proceso de reforma de la enseñanza de la Matemática. Especialistas de esos países están llegando a la Argentina para explicar a funcionarios y expertos locales en qué consisten sus métodos. Alejandro Finocchiaro, Ministro de Educación Nacional le dijo a Clarín que "la idea no es comprar modelos enlatados, sino tomar lo mejor de los países con éxito y adaptarlo a la historia pedagógica y la realidad argentina, para así armar el modelo argentino, que luego podremos exportar a otros países del mundo”.

Lo que tomaron de Francia es el Informe con "21 Medidas para la enseñanza de la matemática” que trajo el especialista francés Charles Torossian y que garantiza 
que en tres años los alumnos de Argentina mejorarán los resultados en Matemática (CLARIN 2018).

Algunos días antes, Clarín publicaba :

Maestros bien formados, salarios atados al rendimiento y programas de estudio con un fuerte foco en matemáticas y ciencias son algunas de las recomendaciones que surgieron de un seminario que organizó la Delegación de la Argentina en la Unesco -a cargo de Rodolfo Terragno- que se hizo en París. Expusieron expertos de Singapur, China, Japón, Corea del Sur, Finlandia y Estonia, países que ocupan los puestos más altos en los rankings internacionales sobre calidad educativa. Participaron el Ministro de Educación Alejandro Finocchiaro, otros ministros provinciales y 19 docentes que ganaron el premio "Maestros Argentinos" (CLARIN, 2018).

Tres cuestiones a destacar en las recomendaciones del Seminario organizado por la Delegación de la Argentina en Unesco: Maestros bien formados, salarios atados al rendimiento y programas de estudio con fuerte foco en matemática y ciencias.

Sobre el primero de los aspectos y atendiendo a los bajos resultados de las evaluaciones la estrategia que adopta el gobierno es el "entrenamiento" de líderes (directores y docentes) o "Reskilling para los maestros" (reciclaje, reconversión, adaptación como propone Davos 2018), cuando en realidad se trata de un mecanismo de disciplinamiento de los docentes y de las instituciones escolares en el discurso de las "prácticas innovadoras para la incorporación de tecnologías de información y comunicación", "buenas prácticas" y formación de líderes para llevar adelante las innovaciones. Mientras los salarios de los docentes atados al rendimiento significa pagos por productividad o por resultados, vinculados a los resultados en los operativos de evaluación de aprendizaje. El fuerte énfasis en los contenidos de Matemática y ciencias responde a lo que diferentes autores mencionan como "El mejoramiento de los estudiantes en habilidades y competencias relacionadas con el empleo" (BALL, 1998, p. 122) y ya forma parte de las prioridades curriculares fijadas por las actuales política nacionales.

Durante el Foro Económico Mundial reunido en Davos en enero 2016 el entonces Ministro de Educación Lic. Esteban Bullrich invitó a Sunny Varkey, creador de la Fundación Varkey, a interiorizarse sobre la situación de la educación en Argentina. La Fundación VARKEY se define como

[...] organización sin fines de lucro creada para mejorar los estándares de educación de todo el mundo. La misión de la organización es que todos los niños tengan un gran docente. Trabajamos para desarrollar las capacidades de los docentes y celebramos sus esfuerzos para que sean reconocidos mundialmente (FUNDACIÓN VARKEY, 2019). 
Apenas un par de meses después de la reunión Bullrich-Varkey en Davos, un equipo de la Fundación viajó a Buenos Aires para diseñar un primer programa para el país desde un enfoque colaborativo. En octubre de 2016 comenzó la primera cohorte de capacitación en la provincia de Jujuy a través del Programa de Liderazgo e Innovación Educativa. Tras ese lanzamiento otras tres provincias iniciaron la capacitación de docentes y directores: Salta, Corrientes y Mendoza. Para cada lanzamiento se conformó un nuevo equipo provincial con tutores locales que luego de un riguroso proceso de selección fue capacitado para llevar adelante una tarea que reflejara la dinámica y fuese flexible para responder a las particularidades de cada región, fortaleciendo el diseño curricular y ajustando el enfoque pedagógico.

El Programa de Liderazgo e Innovación Educativa (PLIE) tiene el objetivo de brindar nuevas herramientas y potenciar las capacidades de directores y docentes de escuelas del país sobre temas como: Liderazgo. Consiste en una capacitación intensiva de seis semanas a la que asiste un director acompañado de un docente de la misma institución. Cada módulo ocupa cinco días y se desarrolla en un centro de capacitación especializado donde se trabaja junto a un grupo de expertos locales.

Los participantes son evaluados a través de un portfolio cuyo objetivo es integrar la teoría vista en cada módulo a la realidad de la propia institución. Cada participante irá creando su propia carpeta que es evaluada y retroalimentada por los facilitadores del programa. A su vez, a lo largo de las seis semanas los participantes tienen que realizar un diagnóstico de su institución y diseñar un proyecto de mejora conocido como Proyecto de Innovación Escolar (PIE). Se dedican sesiones específicas para su realización y socialización. La entrega final es evaluada por los facilitadores del programa y su implementación escolar es acompañada por la Fundación.

Actualmente, el PLIE se implementa en Mendoza, Corrientes, Salta y Jujuy con el apoyo del Ministerio de Educación Nacional y de los Ministerios de Educación provinciales. En Jujuy y Mendoza firmaron convenios con la Fundación y el monto que pagó cada provincia con fondos que envía el gobierno nacional es de 5,4 millones de dólares, pagadero en cuotas mensuales de 150.000 dólares entre Diciembre de 2017 y diciembre 2019. Lo llamativo es la cláusula de exclusividad en el contrato que impide que por tres años se realice en todo el territorio nacional otra actividad similar con otros prestadores (FELDFEBER; OTROS, 2018).

Hasta agosto 2019 en el sitio web de la Fundación Varkey se exponían los siguientes resultados: 
- Formación de más de 7100 directores y docentes de 3700 escuelas a través del Programa de Liderazgo e Innovación Educativa.

- El 93\% de quienes asistieron recomendaría el Programa a sus colegas.

- El 90\% de docentes que no asistieron manifiesta haber percibido cambios en su escuela tras la participación de su directivo en el PLIE.

Finalmente, se destaca que el Programa fue tomado como caso de estudio por la Harvard Graduate School of Education y el Harvard Business School para el Certificate of Advanced Education Leadership professional programme (FUNDACIÓN VARKEY, 2019).

La Coalición Latinoamericana para la Excelencia Docente es una red de más de 14 países que reúne a académicos, gestores de políticas educativas y directivos de instituciones vinculadas a educación con el objetivo de mejorar y apoyar la docencia en los países, construyendo una agenda común y coordinando esfuerzos para su implementación. Algunos de los aspectos del diagnóstico que fundamenta la propuesta refieren a:

Cada vez es más evidente que los educadores juegan un papel fundamental para fortalecer el desempeño de los estudiantes, y que elevar la calidad docente es imperativo para mejorar el aprendizaje estudiantil. Varios países en la región han realizado esfuerzos para reformar ciertos aspectos de sus políticas docentes, vinculados con la formación inicial, el desarrollo profesional, la carrera docente y la evaluación. Sin embargo, varios estudios de la muestran bajos niveles de desempeño docente y una necesidad apremiante de fortalecer las prácticas pedagógicas, conocimientos y destrezas de la docencia (FUNDACIÓN VARKEY, 2019).

El primer encuentro de la Coalición Latinoamericana para la Excelencia Docente se celebró en Buenos Aires el 7 y 8 de Febrero de 2019 organizado por el Diálogo Interamericano, Fundación Varkey e Inicia Educación, con el apoyo de la Organización de Estados Iberoamericanos.

\section{Imposible concluir... necesitamos profundizar el debate}

Carter y O’Neill (citado por BALL, 1998, p. 122) distinguen cinco componentes de lo que denominan "la nueva ortodoxia" o "solución de mercado" para cambiar la educación en contextos neoliberales:

1) El mejoramiento de la economía de los países a través del fortalecimiento de los vínculos entre escolaridad, empleo, productividad y comercio.

2) El mejoramiento del desempeño de los estudiantes en competencias relacionadas con el empleo. 
3) El control directo sobre el curriculum y la evaluación;

4) La disminución de los costos de los servicios educativos que asumen los gobiernos;

5) La participación de la comunidad local a través de: a) una mayor intervención de las familias en las decisiones sobre los asuntos de la escuela y, b) la mayor presión del mercado que pueden ejercer los padres a través de la "elección" de escuelas.

En la política educativa de la Alianza Cambiemos estos componentes están presente y son claramente percibidos en los discursos y textos de las políticas educativas que comenzaron a ejecutarse a partir de 2015. A lo largo del artículo se fue ilustrando con discursos y textos la clara orientación de poner al sistema educativo en relación a las demandas del empleo (al menos en el plano discursivo, por cuanto el desempleo fue una de las variables de ajuste de este gobierno) y de los empresarios.

La jerarquización de la evaluación como una herramienta de rendición de cuenta, de vigilancia y verificación de resultados de aprendizaje que el gobierno vincula como necesarios para economías futuras, mercados laborales de producción flexible, nuevas reglas de contratación y con la competencia escolar desde unas lógica de mercado.

La disminución de los costos en educación que asumen los gobiernos se traducen en los recortes presupuestarios y subejecuciones presupuestarias desde 2016 a la fecha concentrados en las políticas socio-educativas que atienden a los sectores mas necesitados.

En la quinta "nueva ortodoxia", estrechamente vinculada a las anteriores, la "elección educativa" y la mayor intervención de los padres en las decisiones de las escuelas está contenida en los documentos del gobierno cuando define como uno de los ejes de la política educativa el compromiso de la familia, de la comunidad y del sector privado en alcanzar mejores logros educativos.

Para el sostenimiento de esas ortodoxias o soluciones de mercado en las políticas educativas los cargos de gestión en áreas estratégicas como educación fueron ocupados por funcionários, en su mayoría CEOs, que venían del campo empresarial pero con un desconocimiento sobre el funcionamiento del Estado. Esto fue el resultado de desplazar a las universidades como agentes prioritarios ${ }^{12}$ en las consultorías y asesoramiento en las políticas públicas y reemplazar a los equipos 
profesionales de los ministerios de educación por funcionarios, sin trayectoria ni formación específica en el campo educativo.

A 13 años de la sanción de la Ley de Educación Nacional precisamos establecer una agenda de investigación sobre estos nuevos procesos privatizadores que vulneran el derecho humano y social a una educación pública, gratuita con calidad socialmente referenciada y brindada por el Estado. Al mismo tiempo, procurarnos nuevas herramientas teóricas, métodos, conceptos y nuevas sensibilidades para trabajar con "redes políticas", como "nueva forma de gobernanza" que "envuelve tipos específicos de relaciones sociales, de flujos y de movimientos altamente complejos para suponen un gran desafio para la investigación” (BALL, 2013, p. 34).

\section{Notas}

1 Ley de los 180 días de clase № 25864/04; Ley de Financiamiento Educativo № 26075/05; Ley de Educación Técnico Profesional № 26058/05; Programa Nacional de Educación Sexual Integral Ley № 26150/06; Ley de Educación Nacional № 26206/06.

2 En el marco de las políticas educativas para la inclusión educativa se desarrollaron un conjunto de planes, programas y proyectos, entre los que destacamos aquéllos destinados al reingreso, la permanencia y el egreso de adolescentes y jóvenes que habían interrumpido su escolaridad secundaria. Como política social, el Programa Asignación Universal por Hijos (AUH) atiende a las familias con hijos menores de 18 años en condiciones de vulnerabilidad social (desocupados, familias con hijos discapacitados, trabajadores informales que perciben bajos ingresos, servicio domésticas, embarazadas, monotributistas sociales) con el propósito de reinsertar a los alumnas y alumnos que habían abandonado la escuela (primaria y secundaria) e incorporar a nuevas poblaciones que no habían llegado a la escuela secundaria.

3 En el período 2004-2015 se crearon un total de diecinueve universidades nacionales atendiendo al criterio de distribución territorial de las universidades y priorizando las provincias o regiones del país que concentran una población con más restricciones socio-económicas y menor acceso a bienes culturales. Es una constatación que la cercanía de la oferta universitaria disminuye los costos privados atrayendo a nuevas poblaciones o primera generación que accede a los estudios universitarios (los que no asistían a la universidad porque no tenían posibilidades de afrontar los gastos para radicarse en otra ciudad, o tienen familiares a cargo, especialmente las mujeres, o porque la universidad aparecía lejana en sus expectativas y mucho menos como un derecho). En el último decenio, la participación en la educación superior del quintil más pobre creció más del $50 \%$ y la relación entre el $20 \%$ más rico que cursa estudios de nivel superior y el $20 \%$ más pobre pasó del 4/5, a menos del 2/1. Argentina es el país con mayor equidad en el acceso a la educación superior: $18,5 \%$ provienen del primer quintil de ingresos y $24,3 \%$ de segundo quintil, lo que sumandos alcanzarían 43\% del total de alumnos (MIRANDA, 2016; PÉREZ RASETTI, 2014; DEL BELLO, 2015).

4 El Programa Conectar Igualdad se diseñó en 2010 para ser implementado en escuelas de enseñanza del nivel medio, de educación técnico profesional, modalidad especial e institutos de formación docente. En 2016 continúa con el nombre de Plan Nacional de Educación Digital y fue transferido de la Administración Nacional de Seguridad Social (ANSES) al Ministerio de Educación con una fuerte disminución presupuestaria, del orden del $43 \%$ del presupuesto en 2018 , lo que significa una pérdida real de - 52,5\%, acumulados de los presupuestos anteriores.

5 En el marco de la centralidad que ocupa la evaluación en las políticas de Cambiemos se implementa a partir de 2016 el Operativo Aprender (que reemplaza al Operativo Nacional de Evaluación(ONE) creado en la década de los noventa, de periodicidad trianual) para evaluaciones anuales de los aprendizajes aplicable a una muestra de alumnos de los niveles primario y secundario, en diferentes grados o años que 
varían en cada operativo. Por primera vez se llevan a cabo evaluaciones a los estudiantes de instituciones de formación docente (Pruebas Enseñar). Si bien hubo intentos de modificar la Ley de Educación Nacional para introducir la difusión pública de resultados de las escuelas, las iniciativas oficiales no prosperaron.

6 En el presupuesto 2018, entre otros programas, se elimina el Programa de Biblioteca del Maestro. El Programa Formación Docente si bien prevé un incremento del 3\%, en la práctica significa una pérdida real de 14,1\%. La subejecución del presupuesto de 2017 afectó programas de alta sensibilidad social como: Progresar; los Planes de Distribución de Libros de Texto, Lectura y Manuales, Colecciones literarias y Plan Nacional de Lectura; cierre de los Programas de Alfabetización de Adultos; disminución de los subsidios a las escuelas en situación de vulnerabilidad; reducción en cantidad de becas para estudiantes de formación docente, de grado y de posgrado, para carreras universitarias prioritarias y para alumnos universitarios de bajos recursos; desfinanciamiento de la Educación Técnica. El área de Infraestructura escolar también se vio afectada por las políticas de ajuste y de subejecución de los fondos destinados al Mejoramiento de la Infraestructura de las Escuelas- en 2016 sólo se realizaron $241.587 \mathrm{~m} 2,65.000 \mathrm{~m} 2$ menos que el año anterior- y en 2017 apenas se construyeron 40.100 metros cuadrados (CTERA, 2018).

7 Reskilling significa reciclaje, reconversión, readaptación profesional.

8 La Confederación de Trabajadores de la Educación de la República Argentina (CTERA) rechazó la creación de la Secretaría de Evaluación Educativa y defendió la creación del Consejo Nacional porque "fue el resultado de importantes debates y para su aprobación se contó con el acuerdo de una amplia mayoría de las representaciones parlamentarias que, en su oportunidad, votaron a favor de contar con esta instancia que permite construir un sistema integral de evaluación, garantizando un proceso democrático de participación de los miembros de la comunidad académica y científica, representantes del Consejo Federal de Educación, del Congreso Nacional, de las organizaciones del trabajo y la producción, y de las organizaciones gremiales docentes" (CTERA, 2016)

9 El Cuarto Estudio Regional Comparativo y Explicativo (ERCE) se aplica en 2018 a estudiantes de $3^{\circ}$ y $6^{\circ}$ año de escuelas primarias de Educación Común de 18 países de América Latina, en las áreas de Lectura, Escritura, Matemática y Ciencias (esta última solo en $6^{\circ}$ año).

${ }^{10}$ Empresas "multilatinas" son definidas como empresas con sede en América Latina y en rápida expansión por todo el planeta (SANCHEZ ZINNY, 2015, p. 55).

${ }^{11}$ El Decreto n⿳ 641/2018 introduce cambios en el Decreto firmado por el Presidente de la Nación Eduardo Duhalde el 19 de junio de 2002, que priorizaba el asesoramiento de profesores universitarios en asuntos de la administración pública. Por el contrario, este gobierno limita la contratación con las universidades de asesorías, auditorías, investigación, así como capacitación y formación y excluye los convenios de asistencia técnica, dejando abierta la posibilidad de contratar nacionales o extranjeras privadas.

\section{Referencias}

AMAR, H. La arquitectura discursiva de la Política Educativa de Cambiemos. Ponencia presentada en el Panel: Las políticas educativas en la era Cambiemos: entre los discursos y las prácticas. XIV CONGRESO DE LA SOCIEDAD ARGENTINA DE ANÁLISIS POLÍTICO (SAAP). Buenos Aires: Universidad Nacional de San Martín, 17 al 20 de Julio de 2019.

ARMIJO CABRERA, E. Micro-política de una escuela marginalizada entre inclusión y estandarización. Universitas, 20, p. 191-209, 2019.

BALL, S. Educação Global S.A. Novas redes políticas e o imaginário neoliberal. Ponta Grossa: Editora Universidade Estadual de Ponta Grossa, 2014.

BALL, S. Novos Estados, nova gobernança e nova política educacional. En Apple, M.; Ball, S.; Gandin, L.(org.) Sociología da Educação. Analise Internacional. Porto Alegre: Penso, 2013, p. 177-189. 
BALL, S. Política social y educativa, empresa social, hibridación y nuevas comunidades discursivas. En Propuesta Educativa, Buenos Aires, v. 2, nº 36, año 20, p. 25-34, 2011. Disponible en: http://www.redalyc.org/articulo.oa?id=403041707004. Consultado en: 12/08/2019.

BALL, S.; YOUDELL, D. Privatización encubierta en la educación pública. Instituto de Educación, Universidad de Londres. Internacional de la Educación. V Congreso Mundial, Julio 2007.

BALL, S (1998) Policies/Small World: An introduction to international perspectives in education policy. Comparative Education, v. 34, n. 2, p.119-130

CTERA (2016) La CTERA rechazó la creación por decreto de la Secretaría de Evaluación Educativa. Disponible en: https://agencia-popular.com/2016/04/08/la-ctera-rechazo-la-creacionpor-decreto-de-la-secretaria-de-evaluacion-educativa/. Consultado en: 10/08/2019.

CTERA (2018) Informe Analítico de CTERA, Proyecto de Presupuesto Educativo para el año 2018. Disponible en: https://www.ctera.org.ar/index.php/educacion/investigacion/item/2858preocupa-el-ajuste-en-educacion-informe-analitico-de-ctera-proyecto-de-presupuesto-educativopara-el-ano-2018. Consultado el 10/08/2019.

DEL BELLO, J. II Agenda de políticas universitarias. In C. MARQUIS. La Agenda Universitaria II. Propuestas de Políticas Públicas. Buenos Aires: Ediciones Universidad de Palermo, 2015, p. 43-78,

DIARIO CLARIN. Buenos Aires, 12/04/2018; 29/05/2018; 21/05/2019.

EL PAIS DIGITAL. Madrid, 30/05/2018

DIARIO INFOBAE. Buenos Aires, 12/06/2019.

DIARIO LA NACIÓN, 31/01/2018; 21/07/2016.

FELDFEBER, M., et al. (2018) La privatización educativa en Argentina. Buenos Aires: Instituto de Investigaciones Pedagógicas Marina Vilte, Ctera, 2018.

FUNDACIÓN SUNNY VARKEY. Disponible en: https://www.fundacionvarkey.org/. Consultado en: $14 / 08 / 2019$.

GARCIA DELGADO, D.; GRADIN, A. (Comp.). El neoliberalismo tardío. Teoría y Praxis. Documento № 5. Buenos Aires: Flacso, 2017.

GOROSTIAGA, J. El discurso oficial sobre calidad educativa en la gestión Cambiemos. Ponencia presentada en el XIV Congreso de la Sociedad Argentina de Análisis Político. Buenos Aires: Universidad Nacional de San Martín, 17 al 20 de julio de 2019.

LAVAL, C. (2018) Educación Superior en el siglo XXI: ¿bien público o mercancía? En Jornadas de Reflexión sobre tendencias en la educación superior. Uruguay: Universidad de la República, p. 39-58.

MIRANDA, E. El derecho a la universidad en Argentina. Una mirada desde la igualdad y el reconocimiento del otro. Sisyphus Journal of Education, Lisboa, vol. 6, issue 01, p. 79-96, 2018. Disponible en: http://revistas.rcaap.pt/sisyphus/issue/view/816. Consultado 30/07/2019. 
MIRANDA, E. SALTO, D. Dinámicas público-privadas en el posgrado en Argentina: redefiniciones de las tradicionales fronteras en la educación superior. En Peroni, V. (Coord.) Diálogos sobre as redefinições no papel do Estado e nas fronteiras entre o público e o privado na educação, São Leopoldo: Oikos, 2015, p. 276-295.

MIRANDA, E. De la selección a la universalización. Los desafíos de la obligatoriedad de la educación secundaria. Espacios en Blanco. Tandil (Provincia de Buenos Aires), Revista de Educación, núm. 23, junio, p. 9-32, 2013. Disponible en: http://www.redalyc.org/articulo. oa?id=384539805002. Consultado en: 15/08/2019.

PÉREZ RASETTI, C. La expansión de la educación universitaria: Políticas y Lógicas, Integración y conocimiento. Revista del Núcleo de Estudios e Investigaciones en Educación Superior del MERCOSUR, (2), p. 8-32, 2014.

PRESIDENCIA DE LA NACIÓN, JEFATURA DE GABINETE DE MINISTROS, Educación y trabajo: Diagnóstico. Buenos Aires, 2017.

PROYECTO EUTOPIA. La escuela se reinventa. Disponible en https://www. fundaciontelefonica.com.ar/proyecto-eutopia-la-escuela-se-reinventa/. Consultado en: $15 / 08 / 2019$.

RIZVI, F y LINGARD, B. Políticas educativas en un mundo globalizado, Madrid: Morata, 2013.

SANCHEZ ZINNY, G. Educación 3.0: la batalla por el talento en América Latina. Bogotá: Carvajal Soluciones Educativas, 2015.

UNICEF (2019). Políticas educativas para transformar la educación secundaria. Estudio de Casos provinciales a nivel provincial. Primera etapa. Buenos Aires: Flacso/Unicef Argentina. https://www.unicef.org/argentina/sites/unicef.org.argentina/files/201804/Educacion_UNICEF_ Flacso_PoliticasEducativas.pdf. Consultado en: 15/08/2019.

VERDÚN, N.; FOURÉS, C.; CAPUANO, A.; AGUIAR, D. Configuraciones escolares y tic en la educación media. El Programa Conectar Igualdad en tres provincias de Argentina (2011-2012), En Estudios de Comunicación y Política Número 34, p.105-115, 2014. Disponible en: http:// version.xoc.uam.mx. Consultado en: 12/08/2019.

VERGER, A.; PARCERISA, Ll. Globalización, rendición de cuentas y gobernanza educativa: análisis de un fenómeno en expansión. En Con-Ciencia Social (segunda época),1, 59-73, 2018.

VISACOVSKY, N. Enseñar e Investigar Política Educativa en la era Cambiemos (2015-2019): ¿Cómo dimensionar lo que está sucediendo? Ponencia presentada en el Panel: Las políticas educativas en la era Cambiemos: entre los discursos y las prácticas. XIV Congreso de la Sociedad Argentina de Análisis Político (SAAP 2019). Buenos Aires: Universidad Nacional de San Martín, 17 al 20 de Julio de 2019. 